\title{
A Comparison of Pseudo-Bayesian and Joint Maximum Likelihood Procedures for Estimating Item Parameters in the Three-Parameter IRT Model
}

\author{
Gary Skaggs \\ Fairfax County, Virginia Public Schools \\ José Stevenson \\ Montgomery County, Maryland Public Schools
}

\begin{abstract}
This study compared pseudo-Bayesian and joint maximum likelihood procedures for estimating item parameters for the three-parameter logistic model in item response theory. Two programs, ASCAL and LOGIST, which employ the two methods were compared using data simulated from a three-parameter model. Item responses were generated for sample sizes of 2,000 and 500 , test lengths of 35 and 15, and examinees of high, medium, and low ability. The results showed that the item characteristic curves estimated by
\end{abstract}

\begin{abstract}
the two methods were more similar to each other than to the generated item characteristic curves. PseudoBayesian estimation consistently produced more accurate item parameter estimates for the smaller sample size, whereas joint maximum likelihood was more accurate as test length was reduced. Index terms: ASCAL, item response theory, joint maximum likelihood estimation, LOGIST, parameter estimation, pseudoBayesian estimation, three-parameter model.
\end{abstract}

Item response theory (IRT) attempts to model the probability of an examinee's response to a test item as a function of the parameters of the item and the ability or trait level $(\theta)$ of the examinee on the trait measured by the item. IRT models differ in the way item parameters are specified and the way examinee $\theta$ interacts with these parameters. Models have been proposed for a variety of item types and underlying $\theta s$, or dimensions, needed to respond in a certain way to the item. However, the overwhelming emphasis in research has been on unidimensional (i.e., single underlying $\theta$ ) models for dichotomously scored items. Of these models, the most popular have been the Rasch, or one-parameter logistic, model and the three-parameter model. Although these models have different theoretical origins (Birnbaum, 1968; Rasch, 1960/1980), the Rasch model can be subsumed mathematically by the three-parameter model. For this reason, the focus on parameter estimation is typically on the three-parameter model.

Psychometric models based on IRT have been widely used in the past two decades to address a variety of measurement problems and applications. These include equating tests, assessing item bias, deriving scales, and developing computerized adaptive tests. Although IRT models have gained some measure of acceptance, all applications depend on the estimation of item parameters. Until recently, LOGIST (Wingersky, Barton, \& Lord, 1983) has been the only widely available program for estimating parameters for the three-parameter model. It has been used in a wide variety of research studies. However, several

APPLIED PSYCHOLOGICAL MEASUREMENT

Vol. 13, No. 4, December 1989, pp. 391-402

(C) Copyright 1989 Applied Psychological Measurement Inc.

0146-6216/89/040391-12\$1.85 
problems have been noted in its use. These include estimating discrimination and lower asymptote parameters (Baker, 1983; Lord, 1984), the need for large data matrices (Hulin, Lissak, \& Drasgow, 1982), and considerable computer costs.

LOGIST uses what is referred to as a joint maximum likelihood approach to estimate item and $\theta$ parameters. In recent years, alternative approaches have been developed, including marginal maximum likelihood (Mislevy \& Bock, 1982), Bayesian estimation (Swaminathan \& Gifford, 1983), and pseudoBayesian estimation (Vale \& Gialluca, 1985); the latter is the focus of this study. Although some of these methods may prove to be superior to joint maximum likelihood, they have not yet been widely investigated or accepted. Joint maximum likelihood continues to be the standard procedure for many IRT applications and research studies. For a more thorough review of estimation procedures, refer to Baker (1987).

This study examined what is probably the least well known of the new approaches, pseudo-Bayesian estimation, as exemplified by the program ASCAL (Assessment Systems Corp., 1986). According to Vale and Gialluca (1985), ASCAL was modeled after LOGIST and was intended to provide similar results. The program, like LOGIST, is based on a pseudo-maximum likelihood algorithm where item and $\theta$ parameters are estimated in alternate stages. Unlike LOGIST, convergence is achieved by postulating prior distributions for $\theta$, item discrimination, and item lower asymptotes. Swaminathan and Gifford (1985) observed that as the number of items and examinees increased, Bayesian and joint maximum likelihood estimates became more similar. Their estimates differed the most when using small data matrices, a situation known to cause problems of convergence for joint maximum likelihood. This study therefore compared the two estimation methods - as exemplified by LOGIST and ASCAL - under conditions which have been known to cause difficulty for LOGIST, namely short tests and small samples.

In the only other ASCAL evaluation study known to the present authors, Vale and Gialluca (1988) simulated three tests that differed according to the range and level of difficulty and the level of discrimination. Three sample sizes-500, 1,000, and 2,000-were used, all drawn from a standard normal distribution. Estimates for LOGIST were produced only for the largest sample size. Their results suggested that ASCAL and LOGIST produced estimates of equal quality and that ASCAL produced more accurate estimates than two other estimation procedures for smaller sample sizes.

This study expanded on the work of Vale and Gialluca by comparing item parameter estimates from ASCAL and LOGIST for combinations of smaller sample sizes and shorter test lengths. In addition, the $\theta$ level of the sample was manipulated so that average $\theta$ was less than, equal to, or greater than the average item difficulty.

\section{Overview of ASCAL}

ASCAL is described as a "pseudo-Bayesian estimation process"' (Vale \& Gialluca, 1985). The program estimates item and $\theta$ parameters by maximizing a likelihood function in which prior distributions have been included for $\theta$, item discrimination $(a)$, and item lower asymptote parameters $(c)$. The prior distribution for $\theta$ is a standard normal distribution. For the $a$ and $c$ parameters, the specified priors are symmetric beta distributions. The criterion likelihood function is

$\mathrm{L}_{\mathrm{ASCAL}}=\prod_{i}^{N} \prod_{g}^{n_{i}} P_{g}\left(\theta_{i}\right)^{v_{s}} Q_{g}\left(\theta_{i}\right)^{1-v_{g i}} \Phi\left(\theta_{i}\right) f\left(a_{g}, 3,3, .3,2.6\right) f\left[c_{g}, 5,5,-.05,(.05+2 / K)\right]$,

where $\quad N$ is the number of examinees,

$n_{i}$ is the number of items answered by examinee $i$,

$P_{g}\left(\theta_{i}\right)=c_{g}+\frac{1-c_{g}}{1+\exp \left[-1.7 a_{g}\left(\theta_{i}-b_{g}\right)\right]}$, 
$Q_{g}\left(\theta_{i}\right)=1-P_{g}\left(\theta_{i}\right)$

$v_{g i}=\left\{\begin{array}{l}1 \text { if the response to item } g \text { is correct, } \\ 0 \text { if the response is incorrect, } \\ 1 / K \text { otherwise, where } K \text { is the number of alternatives; }\end{array}\right.$

$\Phi$ is the standard normal density function, and

$f(x, r, s, j, k)$ is the Bayesian prior for parameter $x(a$ or $c)$, with upper and lower limits $k$ and $j$, respectively, and beta function parameters $r$ and $s$.

For the $a$ parameter, $r=s=3.0$ with upper and lower bounds of 2.6 and .3 , respectively. For the $c$ parameter, $r=s=5.0$ with a lower bound of 0.0 and an upper bound of $2 / K$, where $K$ is the number of alternatives. There is no prior distribution for the $b$ parameter, but difficulties $(b)$ are restricted to a range of \pm 3.0 .

In contrast to the above function, LOGIST employs the following likelihood function:

$\mathrm{L}_{\mathrm{LOGIST}}=\prod_{i}^{N} \prod_{g}^{n_{i}} P_{g}\left(\theta_{i}\right)^{v_{s}} Q_{g}\left(\theta_{i}\right)^{1-v_{g} i}$.

This likelihood function is similar to the one employed by ASCAL but differs in its lack of specification of prior distributions. Estimates of $a$ and $c$ are limited only by maximum and minimum values.

To obtain parameter estimates which maximize the log of Equation 1, ASCAL employs an algorithm similar to LOGIST where $\theta$ and item parameters are estimated alternately with the estimates of the former serving as an anchor for the estimates of the latter, and vice-versa. Initial $\theta$ estimates are based on a transformation of raw formula scores. Initial $a$ and $b$ parameter estimates are computed from heuristic procedures described by Jensema (1976). The $c$ parameters are initially set to the reciprocal of the number of alternatives for each item. Once $\theta$ s are estimated, they are grouped into 20 fractiles to speed computation. Item parameters are then estimated until convergence is achieved. The sequence of $\theta$ estimation, $\theta$ grouping, and item parameter estimation is repeated until the item parameters converge or the maximum number of iterations has been reached.

ASCAL does not provide many of the options and outputs available from LOGIST. For example, although omitted and not-reached responses are treated in a manner similar to LOGIST, ASCAL has no editing capabilities for excluding cases. There is a Pearson chi-square statistic for testing model fit for each item, but there are no formulations for standard errors of estimates. The user can specify the maximum number of iterations, but not convergence criteria or other aspects of the iteration process. The capacity of the system is limited to 5,000 examinees and 100 items.

Given their similarities, ASCAL and LOGIST would be expected to produce similar estimates for reasonably large datasets. Because of the use of prior distributions, ASCAL should be more accurate for smaller datasets.

\section{Method}

This study compared the accuracy of estimating item characteristic curves (ICCS) under a variety of conditions that have been generally considered to be less than optimal for accurate estimation.

\section{Generation of Item Responses}

Item response $(0,1)$ data were generated from a three-parameter logistic function in which the probability of a correct response to item $g$ is a function of the item's $b, a$, and $c$ parameters, and the 
examinee's $\theta$. It has the following form:

$P_{g}(\theta)=c_{g}+\frac{1-c_{g}}{1+\exp \left[-1.7 a_{g}\left(\theta-b_{g}\right)\right]}$.

For an examinee's response to a single item, the probability in Equation 6 was determined based on known item and $\theta$ parameters. This probability was then compared to a random number drawn from the $(0,1)$ uniform distribution. If the probability was less than the random number, the item was scored as incorrect. Otherwise, the item was scored as correct. The GGUBS (IMSL, 1980) generator was used to generate the random numbers.

\section{Item and $\theta$ Parameters}

For all of the cases studied, $b$ s were uniformly distributed across a range of -2.0 to 2.0 logits, $a$ s were uniformly distributed between .4 and 1.2, and $c$ s were uniformly distributed across a range of .1 to .3. There were three independent variables in this study: sample size, test length, and level of examinee $\theta$.

Item $b s$ were chosen to simulate tests which span a relatively wide range of difficulty. Uniform distributions for the $a$ and $c$ parameters were chosen to simulate tests in which these parameters may vary considerably (e.g., criterion-referenced tests). For such tests, the basis for item selection is more likely to depend on content considerations than on the ability of the item to discriminate between examinees. The distributions of $a$ and $c$ parameters in this study therefore differed slightly from the priors assumed by ASCAL and may have actually favored LOGIST.

Research with LOGIST (Hulin et a1, , 1982) suggested that a minimum of 30 items and 1,000 examinees per item is needed for accurate recovery of generated ICcs. Sample sizes less than 1,000 and tests shorter than 30 items resulted in substantially less accurate estimation. For the present study, sample sizes of 2,000 and 500 and test lengths of 35 and 15 were selected to be above and below the recommended sizes for reasonable estimation.

Three levels of mean $\theta$ were studied: $-1.0,0.0$, and 1.0. In all cases, $\theta$ s were generated from a unit normal distribution. All $\theta$ s were generated using the GGNML (IMSL, 1980) generator. This distribution of $\theta$ fit the prior for ASCAL and was consistent with the metric used by LOGIST. Given that the mean item $b$ was always 0.0 , these levels corresponded to examinee samples of low, medium, and high $\theta$ relative to the test. When mean $\theta$ was 0.0 , the difficulty of the test was perfectly matched to the ability of the sample, the most ideal situation for parameter estimation.

Many tests, however, are designed to be relatively easy or difficult for the target population. Masterylevel achievement tests, for example, typically have a much higher proportion correct than .5. On the other hand, some selection tests are intended to discriminate more highly at the upper end of the $\theta$ distribution. However, very easy tests may provide little information to estimate $c$ parameters. Neither easy nor difficult tests may provide adequate information to estimate $a$ parameters at the opposite end of the scale.

Thus, there were two sample sizes, two test lengths, and three levels of examinee $\theta$ for a total of 12 cells in the design. All LOGIST runs were done on a Unisys 1100/90 computer. All ASCAL runs were done on an IBM PC-XT, PC-AT, or PS/2 Model 70. All microcomputers were equipped with math co-processors. In running LOGIST, all of the default options were used, except that $\theta$ grouping was employed to speed computation. Convergence was typically achieved in 20 to 25 stages for simulated data. For ASCAL, the larger datasets took about 1 hour to run and the smaller ones about 30 minutes, using the PS/2.

At the outset, it was found that a single discrepant item influenced the magnitude of the results to a great extent and resulted in values that were occasionally difficult to interpret. To achieve some stability 
in the findings, each cell based on 15 items was replicated five times and each cell based on 35 items was replicated twice. The replications were combined within each cell so that summary statistics for 15 item tests were actually based on 75 items, and those for 35-item tests were based on 70 items.

\section{Evaluation of Estimation}

For each of the generated datasets, the recovered ICCS for LOGIST and ASCAL estimates were compared to the generated ICCS and to each other using a root mean square error (RMSE) statistic. In addition, differences between the individual parameter estimates were compared using correlations and signed and unsigned differences.

The RMSE statistic is an approximation of the area between ICCs and has the following form:

RMSE $=\left\{\frac{1}{201} \sum_{i=1}^{201}\left[\hat{P}_{g}\left(\theta_{i}\right)-P_{g}\left(\theta_{i}\right)\right]\right\}^{1 / 2}$.

The summation in Equation 7 is across a range of $\theta$ from -3.0 to 3.0 logits in increments of .03 . An RMSE index was computed for each item. When comparing an estimated ICC to a generated ICC, it was first necessary to transform the estimated item parameters to the metric of the generated ICC. This was done by equalizing the means and standard deviations of item difficulties. There are admittedly superior methods for scale transformation (e.g., Stocking \& Lord, 1983), but they could not be used in this study because ASCAL does not provide standard errors for the estimates. Correlations and differences were likewise calculated after transformation to the generated metric.

\section{Results}

\section{Recovery of $\mathrm{HCCS}$}

Average RMSEs for the various combinations of sample size, test length, and sample $\theta$ level are shown in Table 1. Each entry in the table was calculated by averaging the RMSEs for the 70 or 75 items in each cell of the design. The average RMSEs in Table 1 compare ICCS estimated by ASCAL and LOGIST individually to the generated ICCs, and compare estimated ICCs from the two programs to each other (ASCAL/LOGIST).

As expected, estimation was best for both programs for samples of 2,000 and tests of 35 items, and was less accurate when either sample size or test length was reduced. In addition, estimation was predictably better for both programs for samples of medium $\theta$ levels, that is, where sample $\theta$ was matched to the difficulty level of the test. Estimation accuracy was somewhat lower when sample $\theta$ s were lower or higher than the difficulty level of the test.

In general, the results in Table 1 suggest that the two estimation strategies produced very similar ICCS once item parameter estimates were transformed to the same metric. Average RMSEs between the two programs were smaller than those between estimated and generated ICCs in 11 of the 12 cells in the design for ASCAL and in 10 of the 12 cells for LOGIST.

It is apparent from Table 1 that for sample sizes of 2,000 and tests of 35 items, item parameter estimates from the two programs are of comparable quality, a finding consistent with that of Vale and Gialluca (1988). For sample sizes of 500, however, ASCAL produced consistently more accurate estimates than LOGIST. However, for test lengths of 15 items, ASCAL's estimates were not improved by increased sample size and in fact were less accurate than LoGisT's for samples of 2,000. This finding is illustrated more clearly in Figure $\mathbb{1}$. 
Table 1

Average and Standard Deviation of Root Mean Square Errors (RMSEs) Between True and Estimated ICCs for Tests of 35 and 15 Items in Length and Samples of $N=2,000$ and $N=500$ and for Low, Medium, and High $\theta$ Groups

\begin{tabular}{|c|c|c|c|c|c|c|c|}
\hline \multirow{2}{*}{$\begin{array}{c}\theta \\
\text { Group }\end{array}$} & & \multicolumn{3}{|c|}{35 Items } & \multicolumn{3}{|c|}{15 Items } \\
\hline & & ASCAL & LOGIST & $\begin{array}{l}\text { ASCAL/ } \\
\text { LOGIST }\end{array}$ & ASCAL & LOGIST & $\begin{array}{l}\text { ASCAL/ } \\
\text { LOGIST }\end{array}$ \\
\hline \multicolumn{8}{|c|}{$N=2,000$} \\
\hline Low & $\begin{array}{l}\text { Ave. } \\
\text { SD }\end{array}$ & $\begin{array}{l}.039 \\
.021\end{array}$ & $\begin{array}{l}.040 \\
.020\end{array}$ & $\begin{array}{l}.024 \\
.023\end{array}$ & $\begin{array}{l}.059 \\
.034\end{array}$ & $\begin{array}{l}.049 \\
.022\end{array}$ & $\begin{array}{l}.043 \\
.026\end{array}$ \\
\hline Med. & $\begin{array}{l}\text { Ave. } \\
\text { SD }\end{array}$ & $\begin{array}{l}.037 \\
.028\end{array}$ & $\begin{array}{l}.031 \\
.014\end{array}$ & $\begin{array}{l}.024 \\
.025\end{array}$ & $\begin{array}{l}.058 \\
.042\end{array}$ & $\begin{array}{l}.043 \\
.019\end{array}$ & $\begin{array}{l}.048 \\
.027\end{array}$ \\
\hline High & $\begin{array}{l}\text { Ave. } \\
\text { SD }\end{array}$ & $\begin{array}{l}.039 \\
.021\end{array}$ & $\begin{array}{l}.036 \\
.018\end{array}$ & $\begin{array}{l}.044 \\
.017\end{array}$ & $\begin{array}{l}.065 \\
.043\end{array}$ & $\begin{array}{l}.057 \\
.024\end{array}$ & $\begin{array}{l}.052 \\
.030\end{array}$ \\
\hline$N=500$ & & & & & & & \\
\hline Low & $\begin{array}{l}\text { Ave. } \\
\text { SD }\end{array}$ & $\begin{array}{l}.047 \\
.036\end{array}$ & $\begin{array}{l}.057 \\
.035\end{array}$ & $\begin{array}{l}.037 \\
.035\end{array}$ & $\begin{array}{l}.063 \\
.032\end{array}$ & $\begin{array}{l}.076 \\
.037\end{array}$ & $\begin{array}{l}.054 \\
.032\end{array}$ \\
\hline Med. & $\begin{array}{l}\text { Ave. } \\
\text { SD }\end{array}$ & $\begin{array}{l}.036 \\
.019\end{array}$ & $\begin{array}{l}.045 \\
.022\end{array}$ & $\begin{array}{l}.025 \\
.014\end{array}$ & $\begin{array}{l}.053 \\
.025\end{array}$ & $\begin{array}{l}.067 \\
.030\end{array}$ & $\begin{array}{l}.049 \\
.024\end{array}$ \\
\hline High & $\begin{array}{l}\text { Ave. } \\
\text { SD }\end{array}$ & $\begin{array}{l}.050 \\
.023\end{array}$ & $\begin{array}{l}.066 \\
.034\end{array}$ & $\begin{array}{l}.035 \\
.019\end{array}$ & $\begin{array}{l}.060 \\
.038\end{array}$ & $\begin{array}{l}.064 \\
.034\end{array}$ & $\begin{array}{l}.049 \\
.028\end{array}$ \\
\hline
\end{tabular}

Figure 1

Average RMSE for ASCAL and LOGIST for $N=500$ and 2,000 and $k=15$ and 35

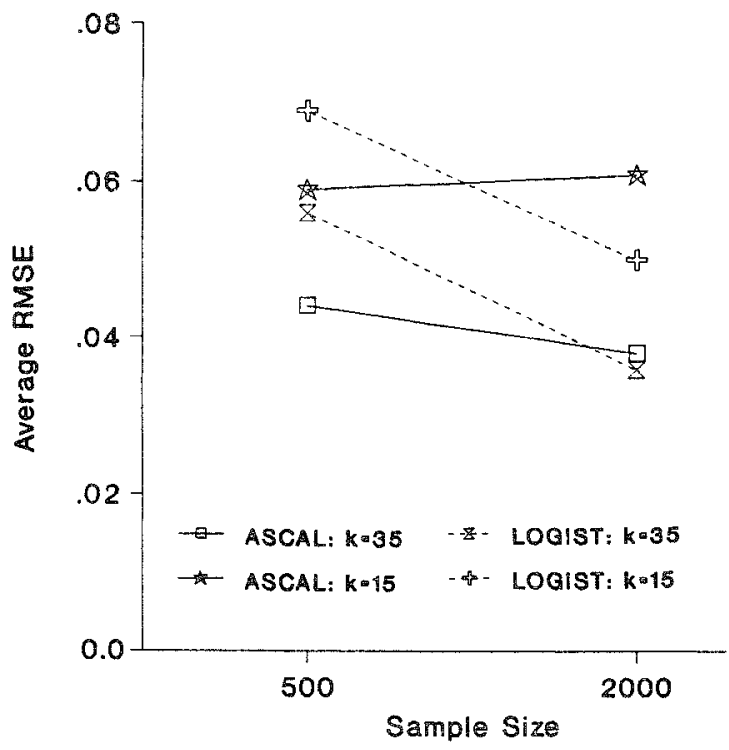


The points in Figure 1 were determined by averaging the RMSEs across all three $\theta$ levels for each combination of sample size and test length. As can be seen in the figure, the estimates for ASCAL, though superior to LOGIST'S for samples of 500, do not markedly change in accuracy between the two sample sizes. Estimation was actually slightly better for samples of 500 for tests of 35 items. Considering the variation in RMSES across the replications within each cell, this difference appears to be well within chance level. In other words, reducing sample size from 2,000 to 500 did not markedly weaken the estimates. The quality of estimates produced by LOGIST, on the other hand, was highly susceptible to reductions in both test length and sample size, a finding reported previously by Hulin et al. (1982).

\section{Correlations Between Estimated and Generated Item Parameters}

Correlations between estimated and generated item parameters are shown in Table 2. In general, the correlations are much less well ordered than average RMSEs. Many correlations, for example, are higher

Table 2

Correlations Between True and Estimated Item Difficulty, Discrimination, and Lower Asymptote Parameters for Samples of 2,000 and 500 and for Low, Medium, and High $\theta$ Groups

\begin{tabular}{|c|c|c|c|c|c|c|}
\hline \multirow[b]{2}{*}{$\begin{array}{l}\text { Parameter } \\
\text { and } \theta \text { Group }\end{array}$} & \multicolumn{3}{|c|}{35 Items } & \multicolumn{3}{|c|}{15 Items } \\
\hline & ASCAL & LOGIST & $\begin{array}{l}\text { ASCAL/ } \\
\text { LOGIST }\end{array}$ & ASCAL & LOGIST & $\begin{array}{l}\text { ASCAL/ } \\
\text { LOGIST }\end{array}$ \\
\hline \multicolumn{7}{|c|}{$\begin{array}{l}\text { Item Difficulty } \\
\qquad N=2,000\end{array}$} \\
\hline Low & .99 & .98 & .99 & .98 & .98 & .99 \\
\hline Mediun & .98 & .99 & .99 & .99 & .99 & .99 \\
\hline High & .99 & .99 & .99 & .98 & .98 & .99 \\
\hline \multicolumn{7}{|l|}{$N=500$} \\
\hline Low & .96 & .94 & .97 & .97 & .93 & .96 \\
\hline Medium & .98 & .96 & .98 & .99 & .95 & .97 \\
\hline High & .98 & .95 & .99 & .98 & .96 & .98 \\
\hline \multicolumn{7}{|c|}{$\begin{array}{l}\text { Item Discrimination } \\
\qquad N=200\end{array}$} \\
\hline Low & .66 & .74 & .79 & .66 & .72 & .71 \\
\hline Medium & .75 & .89 & .88 & .79 & .80 & .74 \\
\hline $\begin{array}{c}\text { High } \\
N=500\end{array}$ & .73 & .89 & .89 & .59 & .64 & .74 \\
\hline Low & .55 & .42 & .72 & .65 & .54 & .75 \\
\hline Medium & .63 & .54 & .86 & .68 & .55 & .81 \\
\hline High & .60 & .58 & .88 & .53 & .54 & .76 \\
\hline \multicolumn{7}{|c|}{$\begin{array}{l}\text { Lower Asymptote } \\
\qquad N=2,000\end{array}$} \\
\hline Low & .55 & .56 & .80 & .59 & .59 & .85 \\
\hline Medium & .61 & .51 & .77 & -.01 & .32 & .54 \\
\hline High & .55 & .46 & .66 & .02 & .13 & .48 \\
\hline$N=500$ & & & & & & \\
\hline Low & .42 & .33 & .72 & .53 & .49 & .79 \\
\hline Medium & .36 & .16 & .73 & .65 & .45 & .64 \\
\hline High & .28 & .16 & .78 & .19 & .34 & .60 \\
\hline
\end{tabular}


for smaller datasets. Across replications, the variation in coefficients was considerable and was influenced greatly by outliers, that is, one or two items in which a parameter estimate deviated substantially from the generated parameter.

Given these qualifications, several patterns emerged from the values in Table 2. First, all of the correlations for item difficulty were all over .90 . In addition, difficulty estimates from the two programs (ASCAL/LOGIST) also correlated at least .96 with each other.

Correlations were not as strong for the $a$ and $c$ parameters. For item discrimination, correlations were higher for samples of medium $\theta$ and for larger datasets. They were also higher between the two programs than for either program individually. Correlations for ASCAL tended to be smaller than those for LOGIST for samples of 2,000 and higher for samples of 500, even though the generated distribution of $a$ s did not fit the prior distribution.

For lower asymptotes, correlations were again higher between the two programs than they were for either program individually. Even though LOGIST produces a default estimate for many $c s$ and $c$ values are restricted to a rather narrow range, the estimates of $c s$ produced by the programs were quite similar. The correlations dropped as sample $\theta$ increased, presumably due to the increased scarcity of data at the lower end. This effect seemed more pronounced for ASCAL at the shorter test length.

\section{Accuracy of Item Parameter Estimates}

Average signed and unsigned differences between estimated and generated item as are shown in Table 3. These differences were calculated after transforming the estimates to the same metric. As a

Table 3

Average Unsigned and Signed Differences for ASCAL and LOGIST

Between Generated $a$ and $b$ Parameters and Their Parameter Estimates

\begin{tabular}{|c|c|c|c|c|c|c|}
\hline \multirow{2}{*}{$\begin{array}{l}\text { Sample Size, } \\
\theta \text { Group, } \\
\text { and Program }\end{array}$} & \multicolumn{3}{|c|}{35 Items } & \multicolumn{3}{|c|}{15 Items } \\
\hline & $\frac{\sum|\hat{b}-b|}{k}$ & $\frac{\sum|\hat{a}-a|}{k}$ & $\frac{\sum(\hat{a}-a)}{k}$ & $\frac{\sum|\hat{b}-b|}{k}$ & $\frac{\sum|\hat{a}-a|}{k}$ & $\frac{\Sigma(\hat{a}-a)}{k}$ \\
\hline \multicolumn{7}{|l|}{$N=2,000$} \\
\hline $\begin{array}{l}\text { ASCAL } \\
\text { LOGIST }\end{array}$ & $\begin{array}{l}.14 \\
.17\end{array}$ & $\begin{array}{l}.19 \\
.19\end{array}$ & $\begin{array}{l}.14 \\
.13\end{array}$ & $\begin{array}{l}.18 \\
.17\end{array}$ & $\begin{array}{l}.62 \\
.32\end{array}$ & $\begin{array}{l}.58 \\
.29\end{array}$ \\
\hline $\begin{array}{l}\text { Medium } \\
\text { ASCAL } \\
\text { LOGIST }\end{array}$ & $\begin{array}{l}.15 \\
.13\end{array}$ & $\begin{array}{l}.21 \\
.14\end{array}$ & $\begin{array}{l}.20 \\
.11\end{array}$ & $\begin{array}{l}.16 \\
.15\end{array}$ & $\begin{array}{l}.62 \\
.29\end{array}$ & $\begin{array}{l}.61 \\
.27\end{array}$ \\
\hline \multicolumn{7}{|l|}{ High } \\
\hline ASCAL & .14 & .25 & .24 & .20 & .61 & .58 \\
\hline LOGIST & .15 & .15 & .13 & .19 & .35 & .33 \\
\hline \multicolumn{7}{|l|}{$\begin{array}{c}N=500 \\
\text { Low }\end{array}$} \\
\hline $\begin{array}{l}\text { ASCAL } \\
\text { LOGIST }\end{array}$ & $\begin{array}{l}.24 \\
.30\end{array}$ & $\begin{array}{l}.30 \\
.33\end{array}$ & $\begin{array}{l}.25 \\
.19\end{array}$ & $\begin{array}{l}.23 \\
.32\end{array}$ & $\begin{array}{l}.55 \\
.54\end{array}$ & $\begin{array}{l}.52 \\
.48\end{array}$ \\
\hline \multicolumn{7}{|l|}{ Medium } \\
\hline ASCAL & .19 & .23 & .16 & .14 & .45 & .39 \\
\hline LOGIST & .26 & .29 & .17 & .29 & .46 & .40 \\
\hline \multicolumn{7}{|l|}{ High } \\
\hline ASCAL & .19 & .29 & .25 & .20 & .55 & .50 \\
\hline LOGIST & .28 & .34 & .27 & .24 & .45 & .38 \\
\hline
\end{tabular}


result, average signed differences for item difficulty were necessarily equal to 0 and therefore are not reported in the table.

The results in Table 3 generally support those reported in Table 1 for item difficulty. Average unsigned differences were slightly smaller for ASCAL than LOGIST for samples of 500 and approximately equal for samples of 2,000. Both signed and unsigned differences were smaller for LOGIST than ASCAL for samples of 2,000 and approximately equal for samples of 500. Interestingly, average signed differences were positive for both programs in all conditions, indicating that the âs tended to be larger in magnitude than the generated as. The mean difference in magnitude sometimes exceeded .50 .

It is also interesting to note that for the smaller sample size, the greater accuracy in overall ICC recovery reported in Table 1 for ASCAL was not replicated for item discrimination. Therefore, the greater accuracy of ASCAL for small samples appears to be due to better estimates of item difficulty rather than discrimination. Because there are no priors for item difficulty in either program, better estimates of item difficulty from ASCAL may in turn be due to the presence of a prior distribution of $\theta$.

\section{Distributions of Item Parameter Estimates}

To better understand the nature of the estimates from the two estimation strategies, the first four moments of the distributions of difficulty and discrimination estimates are shown in Tables 4 and 5 , respectively. The values in these tables reflect the original estimates prior to transformation (which altered only the first two moments). The values in Table 4 for both programs are quite similar to those of the

Table 4

Mean, Standard Deviation (SD), Skewness, and Kurtosis for the Distribution of Estimates for Item Difficulty, Based on Generated Item Difficulties with Mean $=0.0, S D=1.2$, skewness $=0.0$, and Kurtosis 1.8

\begin{tabular}{|c|c|c|c|c|c|c|c|c|}
\hline \multirow{2}{*}{$\begin{array}{l}\text { Sample Size, } \\
\theta \text { Group, } \\
\text { and Program }\end{array}$} & \multicolumn{4}{|c|}{35 Items } & \multicolumn{4}{|c|}{15 Items } \\
\hline & Mean & SD & Skew & Rurt & Mean & SD & Skew & Kurt \\
\hline \multicolumn{9}{|l|}{$N=2,000$} \\
\hline \multicolumn{9}{|l|}{ Low } \\
\hline ASCAL & .93 & 1.28 & .06 & 1.68 & .81 & 1.35 & -.14 & 1.76 \\
\hline LOGIST & .90 & 1.31 & .30 & 2.29 & .57 & 1.23 & -.22 & 2.05 \\
\hline \multicolumn{9}{|l|}{ Mediun } \\
\hline ASCAL & .04 & 1.20 & .17 & 2.05 & .09 & 1.25 & .03 & 1.65 \\
\hline LOGIST & -.06 & 1.22 & -.01 & 1.80 & -.03 & 1.23 & -.07 & 1.83 \\
\hline \multicolumn{9}{|l|}{ High } \\
\hline ASCAL & -.85 & 1.20 & -.02 & 1.77 & -.89 & 1.28 & .10 & 1.82 \\
\hline LOGIST & -.98 & 1.22 & .01 & 1.84 & $-\infty .88$ & 1.37 & -.06 & 1.78 \\
\hline \multicolumn{9}{|l|}{$N=500$} \\
\hline \multicolumn{9}{|l|}{ Low } \\
\hline ASCAL & .96 & 1.20 & -.05 & 1.75 & .93 & 1.19 & -.20 & 1.73 \\
\hline LOGIST & .87 & 1.24 & .14 & 2.37 & .73 & 1.32 & .33 & 3.77 \\
\hline \multicolumn{9}{|l|}{ Medium } \\
\hline ASCAL & .08 & 1.11 & -.04 & 1.82 & .00 & 1.15 & -.03 & 1.96 \\
\hline LOGIST & .02 & 1.18 & -.12 & 2.13 & -.12 & 1.33 & -.58 & 2.91 \\
\hline \multicolumn{9}{|l|}{ High } \\
\hline ASCAI & -.90 & 1.25 & -.07 & 1.84 & -.86 & 1.21 & .16 & 1.99 \\
\hline LOGIST & -1.01 & 1.37 & -.30 & 2.25 & -.91 & 1.48 & -.34 & 3.12 \\
\hline
\end{tabular}


Table 5

Mean, Standard Deviation (SD), Skewness, and Kurtosis for the Distribution of Estimates for Item Discrimination, Based on Generated Item Discriminations with Mean $=.8, S D=.25$, Skewness $=0.0$, and Kurtosis $=1.8$

\begin{tabular}{|c|c|c|c|c|c|c|c|c|}
\hline \multirow{2}{*}{$\begin{array}{l}\text { Sample Size, } \\
\theta \text { Group, } \\
\text { and Program }\end{array}$} & \multicolumn{4}{|c|}{35 Items } & \multicolumn{4}{|c|}{15 I tems } \\
\hline & Mean & SD & Skew & Kurt & Mean & SD & Skew & Kurt \\
\hline \multicolumn{9}{|l|}{$N=2,000$} \\
\hline ASCAL & .88 & .30 & .56 & 2.85 & 1.33 & .74 & .45 & 1.65 \\
\hline LOGIST & .85 & .31 & .45 & 2.67 & 1.14 & .45 & .33 & 2.36 \\
\hline \multicolumn{9}{|l|}{ Medium } \\
\hline ASCAL & .99 & .45 & 1.37 & 5.00 & 1.40 & .82 & .32 & 1.37 \\
\hline LOGIST & .89 & .35 & .71 & 3.50 & 1.09 & .42 & .42 & 2.60 \\
\hline \multicolumn{9}{|l|}{ High } \\
\hline ASCAL & 1.04 & .44 & 1.28 & 5.02 & 1.34 & .83 & .53 & 1.49 \\
\hline LOGIST & .92 & .33 & .46 & 2.90 & 1.02 & .38 & .31 & 2.37 \\
\hline \multicolumn{9}{|l|}{$N=500$} \\
\hline \multicolumn{9}{|l|}{ Low } \\
\hline ASCAL & 1.05 & .43 & 1.25 & 4.86 & 1.38 & .63 & .45 & 1.87 \\
\hline LOGIST & .96 & .46 & .75 & 2.75 & 1.22 & .56 & .14 & 1.64 \\
\hline \multicolumn{9}{|l|}{ Medium } \\
\hline ASCAL & 1.04 & .39 & .65 & 2.92 & 1.29 & .65 & .48 & 1.83 \\
\hline LOGIST & .99 & .45 & .60 & 2.77 & 1.13 & .48 & .36 & 2.45 \\
\hline \multicolumn{9}{|l|}{ High } \\
\hline ASCAL & 1.01 & .36 & .85 & 3.55 & 1.35 & .76 & .37 & 1.43 \\
\hline LOGIST & .94 & .38 & .69 & 3.27 & 1.04 & .51 & .61 & 2.36 \\
\hline
\end{tabular}

generated item difficulties, indicating that both programs were reasonably successful in reproducing the dispersion and shape of the distribution of generated item difficulties, namely a uniform distribution between -2.00 and +2.00 .

The values in Table 5 indicate that the means and standard deviations of item discrimination estimates for both programs were higher than those for the generated discriminations. Transformations to the original metric did not alter this result significantly because the standard deviations of $b$ estimates did not differ greatly from those of the generated $b$ s.

Moreover, distributions of item discrimination estimates were also positively skewed and more highly peaked than those for the generated discriminations. In other words, although generated as were uniformly distributed between .4 and 1.2 , some estimates reached the program ceilings of 2.0 or 2.5 . This finding may appear at first glance to be somewhat surprising.

Apparently, both programs encountered items for which estimates were difficult to obtain. The generated ICcs for these items tended to be of extreme difficulty with low discrimination. For such items, the discrimination estimates occasionally reached the ceiling allowed by the program. The fact that all four moments tended to be higher for ASCAL may have been a function of the higher ceiling value permitted in this study. Introducing a prior distribution for item discrimination apparently had little impact on the resulting distribution of estimates. 


\section{Discussion and Conclusions}

The generated item parameters were intended to reflect instruments, such as achievement tests, where a wide range of item difficulty and discrimination could be expected. As a result, the distributions of discrimination and lower asymptote parameters were symmetric but uniform; thus they differed slightly from the priors assumed by ASCAL and may have favored LOGIST to some degree. Different generating distributions of item parameters certainly may affect the accuracy of ASCAL's estimates. Clearly, different priors for item discrimination may improve the accuracy of ASCAL and/or LOGIST, but it is difficult to hypothesize a shape to the prior distribution when the estimates are always positively skewed.

In general, the estimates produced by both approaches were more similar to each other than to the generated parameters. For samples of 2,000 and tests of 35 items, both programs did very well at recovering the true ICCS. The estimates from ASCAL were affected very little by reducing the sample size from 2,000 to 500, whereas LOGIST's estimates were considerably less accurate. Conversely, ASCAL estimates were more adversely affected than LOGIST's when test length was reduced from 35 to 15 items. It is not known at what test lengths between 35 and 15, or at what sample size below 500, the accuracy of ASCAL would begin to decrease. For the combination of samples of 500 and tests of 15 items, ASCAL produced more accurate estimates even though the generated distributions for $a$ and $c$ differed from those assumed by the program.

Both programs did a reasonably good job of recovering the original distribution of item difficulties. For item discrimination, however, both programs tended to produce positively skewed distributions when the generated distribution was uniform. Evidently this was because a relatively small set of ICCs was difficult to estimate in each dataset. In most cases, these items were either of extreme difficulty or low discrimination or both, and the resulting estimates tended to move toward the ceiling allowed by each program. This suggests that the improved accuracy of ASCAL at smaller sample sizes may be due more to the prior placed on $\theta$ than to the priors for the $a$ and $c$ parameters. It would be interesting to examine ASCAL's accuracy when sample $\theta$ is not normally distributed.

Different configurations of item parameters, examinee $\theta$, test length, and sample size could have been chosen, and they may have demonstrated effects not found in this study. The cases and datasets used in this investigation were chosen to represent a reasonable range of conditions under which LOGIST was known to vary in its accuracy, as well as to represent a fair sampling of data problems found in practice.

Based on the results of this study, pseudo-Bayesian estimation provides a reasonable alternative to joint maximum likelihood. The choice between the two programs may ultimately depend on factors in addition to accuracy, such as the cost of equipment and computer time and the type of application (e.g., ASCAL does not output standard errors of estimates). However, the results of this study suggest that when faced with the common situation of having samples as small as 500, pseudo-Bayesian estimation will produce more accurate estimates.

\section{References}

Assessment Systems Corporation. (1986). MICROCAT: A computer program for computerized adaptive testing. St. Paul: Author.

Baker, F. B. (1983). Comparison of ability metrics obtained under two latent trait theory procedures. $A p$ plied Psychological Measurement, 7, 97-110.

Baker, F. B. (1987). Methodology review: Item parameter estimation under the one-, two-, and three-param- eter logistic models. Applied Psychological Measurement, 11, 111-141.

Birnbaum, A. (1968). Some latent trait models and their use in inferring an examinee's ability. In F. M. Lord \& M. R. Novick, Statistical theories of mental test scores (pp. 397-479). Reading MA: Addison-Wesley.

Hulin, C. L., Lissak, R. I., \& Drasgow, F. (1982). 
Recovery of two- and three-parameter logistic item characteristic curves: A monte carlo study. Applied Psychological Measurement, 6, 249-260.

IMSL, Inc. (1980). International mathematical and statistical libraries reference manual. Houston: Author.

Jensema, C. J. (1976). A simple technique for estimating latent trait mental test parameters. Educational and Psychological Measurement, 36, 705-715.

Lord, F. M. (1984). Maximum likelihood and Bayesian parameter estimation in item response theory (ETS Technical Report No. RR-84-30-ONR). Princeton NJ: Educational Testing Service.

Mislevy, R. J., \& Bock, R. D. (1982). BLLOG: Maximum likelihood item analysis and test scoring with logistic models for binary items. Chicago: International Educational Services.

Rasch, G. (1960). Probabilistic models for some intelligence and attainment tests. Copenhagen: Danish Institute for Educational Research. [Expanded edition, University of Chicago Press, 1980.]

Stocking, M. L., \& Lord, F. M. (1983). Developing a common metric in item response theory. Applied Psychological Measurement, 7, 201-210.

Swaminathan, H., \& Gifford, J. A. (1983). Estimation of parameters in the three-parameter latent trait model. In D. J. Weiss (Ed.), New horizons in testing: Latent trait test theory and computerized adaptive testing (pp. 13-30). New York: Academic Press.

Swaminathan, H., \& Gifford, J. A. (1985). Bayesian estimation in the two-parameter logistic model. Psychometrika, 50, 349-364.

Vale, C. D., \& Gialluca, K. A. (1985). ASCAL: A microcomputer program for estimating logistic IRT item parameters (ONR-85-4). St. Paul: Assessment Systems Corporation.

Vale, C. D., \& Gialluca, K. A. (1988). Evaluation of the efficiency of item calibration. Applied Psychological Measurement, 12, 53-67.

Wingersky, M. S., Barton, M. H., \& Lord, F. M. (1983). LOGIST: A computer program for estimating examinee ability and item characteristic curve parameters. LOGIST 5, Version 1. Princeton NJ: Educational Testing Service.

\section{Acknowledgments}

The authors thank Ronald Hambleton and two anonymous reviewers for their helpful suggestions on earlier drafts of this manuscript, and the Computer Science Center at the University of Maryland.

\section{Author's Address}

Send requests for reprints or further information to Gary Skaggs, Office of Research and Evaluation, Fairfax County Public Schools, 7423 Camp Alger Road, Falls Church VA 22042, U.S.A. 